\title{
El oficio profesional de la Sociología y otras profesiones en clave de mujer
}

\author{
$\mathrm{M}^{\mathrm{a}}$ Amparo ALMARCHA BARBADO
}

Universidad de la Coruña

amparo.almarcha@udc.eso

Recibido: Marzo 2014

Aceptado: Mayo 2014

\section{RESUMEN}

Desde la doble perspectiva profesional y docente se trata de analizar las diferencias y desigualdades que acontecen a las mujeres, teniendo en cuenta que se da un cierta superioridad relativa al mérito y al esfuerzo en el terreno educativo. El esfuerzo de las mujeres no se premia en el mercado de trabajo. Hay mayor número de mujeres tituladas en bastantes espacios y disciplinas, pero todavía se ve como algo espectacular que ocupen buenos puestos en la política y como directivas de empresa. El género femenino es un "pequeño" obstáculo para eso; y las desigualdades y exclusiones se producen desde lo académico a lo profesional. Las barreras siguen existiendo desde la docencia, donde las mujeres escalan en medidas bastantes menores las cátedras o las direcciones de facultades, centros o departamentos. Siguen existiendo las tareas domésticas y lo familiar como barrera. Esto es también constante y posible cuando contemplamos lo que ocurre en el desarrollo profesional de la Sociología y la Ciencia Política: se desbocan las cifras a la baja en la participación De las mujeres como catedráticas, directoras de departamento o decanas, entre otros puestos de relevancia. Está claro que la igualdad no es solo una cuestión de leyes, hay mucho más que poner en juego.

Palabras clave: Género, oficio profesional, mérito y esfuerzo, desigualdad y exclusión.

\section{The professional office of Sociology and other professions in women's key}

\begin{abstract}
From the dual professional and teaching perspective, it is a question of analyzing the differences and inequalities that happen to women, taking into account that there is a certain superiority related to merit and effort in the field of education. The effort of women is not rewarded in job market. There are more graduate women in quite a few spaces and disciplines, but the fact that they hold good positions in politics and as company directives it is still seen as something spectacular. The feminine gender is a "small" obstacle for that; also, inequalities and exclusions occur from the academic sphere to the professional one. The barriers continue to
\end{abstract}


exist from teaching, where women scale in much smaller measures the chairs or the addresses of faculties, centers or departments. Domestic chores continue to exist and the family as a barrier. This is also constant and possible when one observes what happens in the professional development of Sociology and Political Science: downward figures increase concerning women's participation as professors, department directors or deans, among other positions of relevance. It is clear that equality is not only a matter of laws, there is much more to bring into play.

Keywords: Gender, professional trade, merit and effort, inequality and exclusion.

\section{INTRODUCCIÓN}

Mi doble perspectiva como profesional y como docente supone tener en cuenta un largo camino, desde mis comienzos en esas tareas a finales de los años 60, teniendo en cuenta el trabajo profesional en el despacho de Sociología, fundado por Amando de Miguel. Anteriormente, también colaboré en Data, como empresa dedicada a los estudios de mercado y sociología aplicada. Tras terminar mis estudios en Psicología decidí marcharme a Estados Unidos a estudiar de manera formal la carrera de Sociología, durante tres cursos (1975-1978), en la Universidad de Yale. Desde aquella etapa el tiempo profesional lo he dedicado desde el curso 1978-1979, que me incorpore a la Universidad de Granada, a dar clase e investigar en distintos centros educativos, pasando por diferentes niveles en el profesorado, desde "PNN", ayudante, titular para terminar en la Universidad de A Coruña, tras mi última oposición como catedrática de Universidad, en Enero de 1995.

Hace un par de meses finalizaba un artículo, una vez más, para una Libro Homenaje; en este caso el homenajeado era Amando de Miguel, uno de mis maestros en la aventura de la Sociología. Para la mayoría de los colaboradores que empezamos a aprender lo que significaba y para qué podía servir la Sociología en aquellos momentos, dejamos a un lado el anacrónico vocablo de oficio, y lo convertimos en "aventura". Explico de manera más amplia en el texto homenaje que tituló "Aquellos días de Serrano 19", la razón importante que se estaba moviendo al cumplir la tarea de hacer sociología:

"Serrano 19" supuso un modo de hacer sociología alejado del modelo academicista tradicional. Desde mi propia mirada yo aprendí antes a trabajar en la ciencia sociológica, y la comprendí más tarde. Conocí a Amando de Miguel en uno de sus oficios, más preciado, enseñar sociología en el aula, cuando él impartía una clase semanal sobre Estructura Social de España para futuros profesionales de Trabajo Social". 1

Hacer sociología, era para muchos de nosotros algo tan novedoso, que nos "enganchaba" y nos permitía trabajar con "toda ilusión". En mi caso concreto,

\footnotetext{
1 "Amparo Almarcha, "Aquellos días de Serrano 19”, en García Sanz, B., Iglesias de Ussel,J., Oltra, B., Martinez Paricio, J., Valles, M., De la sociedad española y otras sociedades. Libro Homenaje a Amando de Miguel, Madrid, CIS, 2013
} 
comencé demasiado pronto en esas tareas. Participé en un estudio de mercados para una conocida marca de refrescos, haciendo entrevistas en la calle. Creo que era el año 1966, casi no sé quién era yo, pero sí tengo el recuerdo del placer de preguntar, de intentar saber sobre la percepción del comportamiento humano, en esas primeras indagaciones, que suponían las preguntas de los cuestionarios que manejábamos. Aquellos "oficios" llenaron el espacio profesional y la formación del día a día que orientaba nuestra participación tanto en la empresa DATA, como más tarde en el despacho profesional ${ }^{2}$ de A. de Miguel:

"Unos años después, en 1969, Amando se separaba de sus colegas de la empresa DATA, y fundaba el despacho de Sociología en el piso $6^{\circ}$ de la calle Serrano 19 en Madrid. En esa nueva andadura le acompañamos sus colaboradores más directos del llamado Departamento de Diseño en Data ${ }^{3}$. La disposición de esa oficina era casi perfecta, para seguir siendo protagonistas, junto a otros nuevos 'becarios' en aquella aventura".

No es demasiado común pero en mi caso la mayoría de mi formación, y preparación académica ha tenido un destino: la sociología como quehacer, bien como práctica profesional, o bien como enseñante en las universidades en las que he participado, en los 35 años que llevo ejerciendo como profesora.

\section{SITUACIÓN GENERAL DE LAS MUJERES EN RELACIÓN CON EL NIVEL DE ENSEÑANZA ADQUIRIDO}

La situación actual en el espacio educativo y en el terreno profesional, o de participación en el mercado de trabajo es, ante todo, diferente y desigual si la comparamos con lo que les pasa, en ese mismo espacio, a los varones.

De forma general, nos encontramos con que el rendimiento educativo en el nivel de las enseñanzas básica y secundaria es bastante desigual. Así, según datos del INE referidos a febrero de 2012, el abandono educativo temprano es una de las tasas más alta en los países de la UE, junto a países como Malta y Portugal. No obstante, las cifras son bastante más altas para los varones:

"En el año 2011, la cifra de España para los varones (31,0\%) duplica la cifra de UE-27 (15,3\%), el valor que alcanza España sólo es superado por Malta (38,9\%)

\footnotetext{
${ }^{2}$ Amparo Almarcha, “Aquellos días... cit, pág 12.

3 El propio Amando relata esa experiencia en un reciente libro, Memorias y desahogos, publicado en 2010 por Infova Ediciones, como ese proceso de cambio contínuo en esos años de crecimiento y difusión del saber sociológico aplicado al estudio de la sociedad. Comenta: "Era una decisión bastante arriesgada, pero entendí que Data debía continuar con estudios de tipo comercial y rentable" (pág. 200). Dicho de otro modo, "los de diseño" éramos un grupo molesto para la empresa, se nos veía como "engreídos investigadores que no producíamos demasiado económicamente valioso".
} 
y es ligeramente superior al de Portugal $(28,2), 4$

La cifra de abandono en el caso de las mujeres fue del 21,9\%, en 2012. Este dato, tan significativo, y que se ha ido reduciendo en los últimos cursos revela esa situación de desigualdad, que podría indicar un mayor rendimiento y adaptación a las mujeres en su conjunto a la disciplina académica, y el hecho de ser más competitivas o bien pensar que el mérito, o el hecho de ser más estudiosas, les puede permitir un mayor éxito social. De igual modo, según la misma fuente:

“En el año 2011, el porcentaje de mujeres de 20 a 24 años que ha completado el nivel de estudios secundarios de $2^{\mathrm{a}}$ etapa $(69,2 \%)$ es superior a la de los varones $(54,5 \%)$. En los últimos años, el porcentaje de población joven que alcanza este nivel educativo se ha mantenido prácticamente constante, tanto en mujeres como en varones". 5

La tasa de superioridad o excelencia que se observa para las mujeres requiere una reflexión y al menos la consideración de que el espíritu de superación y el aprecio por el mérito escolar confronta dos estilos o maneras de ver la vida que son ostensibles. Dependerá de que los varones esperen una mayor compensación por parte del mercado de trabajo, y que necesiten los títulos en menor medida; no así las mujeres que requieren en este ámbito demostrar sus cualidades y posibles titulaciones. Por todo ello, en ese mismo Informe la reflexión es contundente:

"Las tasas brutas de población que se gradúa para el curso 2009-10 son superiores en las mujeres para todos los niveles educativos considerados. La mayor diferencia en puntos porcentuales entre la tasa de mujeres y la de los varones (14,5 puntos) corresponde al nivel educativo de bachillerato/ COU, con una tasa de 56,1 para las mujeres y de 41,6 para los varones, seguido de la tasas brutas de población que se gradúa en Diplomado Universitario y Arquitecto e Ingeniero Técnico (11,4 puntos) con una tasa de 25,4 en mujeres y 14,0 en varones." 6

Por la misma razón, el mayor o mejor rendimiento de las mujeres también se observa en grupos de edades más altas que las del sistema educativo general:

"En todas las convocatorias de 2010-2011 fue mayor el porcentaje de aprobados varones, excepto en la convocatoria de mayores de 45 años, en la que aprobaron un $44,8 \%$ de varones y un $51,1 \%$ de mujeres, respecto del total de presentados. En el alumnado matriculado en estudios universitarios de primer y segundo ciclo (incluidos los estudios de grado) correspondiente al curso 201011, la mayor participación femenina corresponde a diplomaturas con una

4 INE, Hombres y mujeres 2011, Educación, 3.2. Abandono educativo temprano, http://www.ine.es

${ }^{5}$ INE, Hombres y Mujeres, cit., pág. 8

${ }^{6} \mathrm{INE}$, cit., pág. 10 
participación del $68,2 \%$, seguido de las titulaciones dobles $(59,9 \%)$ y de las licenciaturas $(59,8 \%)$ ". 7

Esta superioridad, relativa al mérito y al esfuerzo en el terreno educativo, no tiene la misma dimensión cuando se trata del empleo. Las mujeres se esfuerzan, pero no reciben a cambio. Parece que el mercado de trabajo les da la espalda. Hay algunos datos significativos.

"En España en el año 2007, la brecha de género (varones-mujeres) en las tasas de empleo de la población de 16 y más años alcanzaba un valor de 21,2 puntos. En el periodo 2007-2011 disminuyó 9,3 puntos alcanzando un valor de 12,0 puntos en el año 2011. Por grupos de edad, el mayor descenso en el valor de la brecha de género se produjo en el grupo de 55 a 64 años (11,6 puntos), seguido de la población joven de 16 a 24 años (11,3 puntos) y del grupo de 20 a 64 años $(10,6$ puntos)". 8

En ese mismo ámbito, los datos que siguen a continuación relacionados con el avance de las tituladas, indican que en el año 2010 el número de mujeres graduadas en educación superior era un $37 \%$ superior al número de varones, 137 mujeres por cada 100 varones. Así:

"Esta cifra era del 40,3\% en el año 2007. En la UE-27 para el mismo año, la cifra de mujeres graduadas era un $50,7 \%$ superior al número de varones. En algunos países, el valor de este indicador en el año 2010 era notablemente superior a la media de la UE-27, como el caso de Letonia (248,6 mujeres por cada 100 varones) y de Estonia (235,7 mujeres por cada 100 varones). El valor más bajo correspondía a Austria (106,2 mujeres graduadas por 100 varones)". 9

El avance tan espectacular de las tituladas mujeres puede explicarse suponiendo que, dado que se les hace más difícil insertarse en el mercado de trabajo, "sacan la cabeza" por otros lugares: se titulan. Ahora bien, esas titulaciones son en aquellos espacios o disciplinas, cuya labor o dedicación corresponde a lo que tradicionalmente se espera de las mujeres: la educación de los más pequeños, y el cuidado de los mayores.

"Por ámbito de estudio, el mayor porcentaje de mujeres que se gradúan en educación superior respecto al total de alumnos graduados corresponde al campo de Educación y formación, tanto en España (79,0\%) como en la UE-27 $(78,9 \%)$, seguido a continuación del campo de Salud y bienestar con un 76,6\% en España y un $77,0 \%$ en la UE-27. El menor porcentaje corresponde a

\footnotetext{
${ }^{7}$ INE, cit., pág. 11

${ }^{8}$ INE, Hombres y mujeres, 2010, cit., pág. 16

${ }^{9} \mathrm{INE}$, cit., pag.19
} 
Ingeniería, Manufactura y Sector de la construcción, con un 27,8\% en España y un $26,2 \%$ en la UE- $27 \%{ }^{10}$

\section{EL CONCEPTO DE GÉNERO EN CLAVE SOCIOLÓGICA}

Explicar este tema significa acudir a la clásica fórmula de asimilar a lo genético un carácter inmutable. Por otra parte, lo adquirido es modificable. Sin embargo, conviene recordar que a menudo es más fácil cambiar características genéticas, frente a la construcción histórica, social y cultural. Se ha constatado que existen estructuras más estables o, a veces, rígidas como son las estructuras adquiridas. Desde otro punto de vista, hay que considerar la manera de delimitar cómo se fabrica el constructo género. Se trata de un desarrollo metodológico o bien técnico de sus componentes, yendo del constructo a las dimensiones, a las sub-dimensiones, después a los indicadores, y a las variables componentes. Generalmente, las variables o indicadores fundamentales en el análisis, tienen dimensiones diversas como son la edad, el nivel educativo alcanzado, la ocupación o el nivel de renta, que entre otras pueden delimitar las diferencias que presenta la variable sexo, y añadir o restar significado a las diferencias o desigualdades que se puedan producir entre las personas. ${ }^{11}$

En la actualidad, han surgido formas sociales emergentes respecto al sexo, las conocidas como innovaciones en relación con las ciencias. La innovación con perspectiva de género "se define como el proceso de integrar el análisis de género y sexo en cada fase de la investigación básica y aplicada, con el fin de asegurar la calidad y excelencia de los resultados" ${ }^{12}$. Esta es la perspectiva del trabajo de Inmaculada Perdomo y Ana Puy en relación con áreas de conocimiento más cercanas a las ciencias, la medicina o la ingeniería, y por ello en otra dimensión al análisis que desde el plano sociológico hacemos de la rama de humanidades o ciencias sociales, casi siempre consideradas como menos científicas que las primeras. Se trataría de un modelo para observar las disciplinas desde el amplio marco del conocimiento, desde la óptica clásica de ciencias y letras, y como dos mundos diferentes y enfrentados sobre todo en sus funciones y papeles dentro de la sociedad. Para poder modificar esa situación y romper barreras es preciso reflexionar sobre los aspectos de género en relación con esa posible dualidad del conocimiento. En este mismo sentido estas autoras concluyen:

\footnotetext{
${ }^{10}$ INE, cit., pág., 17

${ }^{11}$ Para comprender el significado de esta relación desigualdad/diferencia podemos adentrarnos en la explicación más biológica de las diferencias de género que plantean Patricia Madoo y Jill Niebrugge-Brantley respecto al hecho de que "las explicaciones biológicas han sido características del pensamiento conservador sobre las diferencias de género" en "Teoría Feminista Contemporánea” (pág. 367) en George Ritzer, Teoría Sociológica Contemporánea, Madrid, McGraw-Hill, 1993, pp. 353-409

12 Perdomo Reyes, Inmaculada y Ana Puy Rodríguez, Género, conocimiento e investigación, Madrid, Plaza y Valdés, 2012, pág. 19
} 
"Las innovaciones de género contribuyen a lograr mayor excelencia en ciencia, medicina e ingeniería, tanto en términos del conocimiento como del personal; conducen a una ciencia y a una tecnología que son responsables en relación al género y tienen como objetivo mejorar las vidas de mujeres y hombres de forma global". 13

Por todo ello, es necesario tener en cuenta, siguiendo a estas mismas autoras, que la mayoría de estos enfoques suponen, por una parte corregir el número de mujeres que participan en campos tales como la ciencia en general, la medicina y la ingeniería; en segundo lugar, cambiar las instituciones de investigación, eliminando barreras y transformando estructuras $\mathrm{y}$, por último, corregir el conocimiento incorporando el análisis de género a la investigación básica y aplicada. Posiblemente este sería también un objetivo dentro de áreas tales como las llamadas ciencias sociales y humanidades donde la presión científica se manifiesta en diferencias tales como el manejo de técnicas estadísticas y programas informáticos que colocan en escalones de importancia y de desarrollo a una buena parte de ellas. Así, las ciencias económicas se enfrentan a la sociología, como la pedagogía lo hace con la psicología. Otra división más sutil, como es el hecho de ser o no aplicadas o servir para la intervención supone un nuevo riesgo en temas de género.

\section{DIFERENCIAS, DESIGUALDADES Y EXCLUSIONES RESPECTO A LA PARTICIPACIÓN EN EL ÁMBITO DEL MUNDO UNIVERSITARIO: ALUMNAS Y DOCENTES}

Nos encontramos, por tanto, ante una nueva manera de mirar la universidad en su composición. El hecho de que se hayan invertido las cifras de participación, sobre todo entre el alumnado en la mayoría de las comunidades y universidades en nuestro país es cuando menos revolucionario. Llevo varios años estudiando la Universidad y he pasado por diversas explicaciones, ninguna de las cuales me convence de modo total, para entender la excelencia de las mujeres y los abultados índices de participación. ¿Son más aplicadas las mujeres, o más dóciles? ¿O son más inteligentes? O bien necesitan demostrar que valen teniendo más y mejores titulaciones, o sólo se "cuelan" en aquellos puestos que otros, varones casi siempre, dejan a un lado. También podría ser que los estudios valen cada menos y exigen menos competitividad. Pero, ¿qué les pasa a los varones? ¿Son más aburridas las aulas para los varones? ¿No les van a valer esos títulos en el mercado? El estudio exige mucha disciplina y es posible que no sean tan capaces como sus compañeras. ¿Pueden heredar o bien ocuparse de negocios familiares antes que sus colegas de pupitre? No parece que sepamos y/o tengamos investigación suficiente en este terreno para poder contestar a estos interrogantes con criterio científico.

${ }^{13}$ Perdomo Reyes, Inmaculada y Ana Puy Rodríguez, cit., pag.21 


\subsection{UNIVERSIDAD-ALUMNADO: COMPOSICIÓN, ACCESO Y SALIDAS}

En décadas pasadas hemos ido constatando el avance de las mujeres llenando literalmente, como media, los pupitres en las universidades. En este sentido, parece que estamos a nivel europeo:

"En el alumnado matriculado en estudios universitarios de primer y segundo ciclo (incluidos los estudios de grado) correspondiente al curso 2010-11, la mayor participación femenina corresponde a diplomaturas con un $68,2 \%$, seguido de las titulaciones dobles $(59,9 \%)$ y de las licenciaturas $(59,8 \%) . " 14$

De igual modo, en el mercado de titulados/as salen malparadas las mujeres. Esto es bastante injusto, y casi nadie se queja ni se reclama a los autoridades por sus políticas públicas que no corrigen esta situación, diríamos casi endémica ¿dónde está el feminismo reclamando un mejor trato para las mujeres en este terreno?

"En el año 2011, un 36,3\% de los varones y un 45,0\% de las mujeres de 30 a 34 años habían alcanzado un nivel de formación correspondiente a educación superior (CINE niveles 5-6). Este porcentaje se ha elevado ligeramente en España desde el año 2007 tanto en el caso de los varones $(34,8 \%$ en 2007 y $36,3 \%$ en 2011) como en el de las mujeres (44,6\% en 2007 y $45,0 \%$ en 2011 )".

Con respecto a lo anterior nada ha variado. Eso no se traduce de forma visible en el aumento de puestos de trabajo en el mercado. Como ya hemos visto anteriormente, los niveles en Educación y formación $(79,0 \%)$ y en Salud y Bienestar $(76,6)$ respecto a los datos globales, demuestran una superioridad en número por parte de las mujeres. Ahora bien, eso no ha modificado los coeficientes de participación en el mercado laboral remunerado. De igual modo, en el año 2010, el número de mujeres graduadas era casi un $40 \%$ más que el de varones. Y esa cifra conservaba esa misma proporción desde 2007.

Nosotros podemos aventurar que ese mercado no va a girar sus puertas de modo más amplio a las mujeres. El mito del "eterno femenino" puede estar en el hecho de que las barreras sean más sutiles y estén colocadas tanto de fuera hacia dentro como en la otra dirección ¿Qué pasa con el trabajo doméstico productivo? ¿Quién realiza las tareas domésticas, tanto las más corrientes, como las más delicadas, tales como son el cuidado de enfermos y mayores? En etapas nuevas de cambio social, con crisis tanto laborales como sociales, ¿quién o quiénes sacrifican su libertad de elección para trabajar fuera del hogar en trabajos remunerados? Presentimos que en muchos casos esos hogares van a sacrificar las posibilidades de bastantes mujeres más preparadas muchas de ellas, y dada la dificultad de pagar por las tareas del hogar a empleadas/os para tal fin, son las propias mujeres las que adoptan el papel de madres, educadoras, cuidadoras de salud, y también como no, de limpiadoras, cocineras, etc. Parece que el

${ }^{14}$ INE, Informe Hombres y mujeres 2011, pág. 28 
sueño de los 90 de "que la presencia en el mercado laboral de mujeres que son madres es imparable" $"$, se ha podido romper a pesar de todos los esfuerzos de conciliación entre vida laboral y vida doméstica. No obstante existe una grave confusión ¿puede pretenderse que la vía doméstica no es también laboral para un gran número de mujeres?

Recientemente otros estudios indican que "existen dos formas opuestas, aunque no contrarias, de enfrentarse al análisis de la realidad de las mujeres en el mercado laboral" $"$.

\subsection{EL DOMINIO DE LO FEMENINO: CARRERAS FEMENINAS Y CARRERAS MASCULINAS}

Los cambios más significativos, en subida de participación o en mantenimiento de cifras (medicina e ingeniería electrónica) son los siguientes:

- El número de graduados superiores en ciencias, matemáticas y tecnología por mil habitantes entre la población de 20 a 29 años es más elevado en los varones, tanto en España como en la UE-27. En el año 2010 en España, el número de graduados varones es de 18,9 por 1.000 habitantes y 8,6 el número de mujeres graduadas.

- En la UE-27 en el año 2010, el valor de este indicador es más bajo tanto para varones $(16,6 \%)$ como para mujeres $(8,3 \%)$.

- Desde el año 2007, en España, el número de mujeres graduadas en ciencias, matemáticas y tecnología ha aumentado ligeramente, tanto en varones como en mujeres, con un valor de $15,3 \%$ en los varones y de $6,9 \%$ en las mujeres en el año 2007. En la UE-27, aunque la tasa también ha aumentado desde el año 2007, en el año 2010 ha descendido, tanto en varones como en mujeres.

- En países de la UE, tales como Finlandia, Eslovaquia y Rumanía se sitúan los tres primeros con las tasas más altas de mujeres graduadas en ciencia, matemáticas y tecnología en el año 2010. Por el contrario, Chipre, Países Bajos y Luxemburgo se sitúan en los tres últimos puestos. España se encuentra justo por encima de la media de la UE-27.

Lo más significativo o interesante de todo este flujo puede ser casi una sorpresa. Se están reduciendo las cifras de participación en el sistema educativo. En sólo un quinquenio la crisis económica cobra sus víctimas. El número de estudiantes se reduce. No hay fondos económicos para "excesos" Es posible que la reducción o

${ }^{15}$ C.Dominguez Alcón, "El futuro de la conciliación entre la vida laboral, familiar y personal: Mujeres, trabajo y calidad de vida" Mimeografiado, pág. 13

${ }^{16}$ Amparo Almarcha Barbado y Benjamín González Rodríguez, "Perfil social de las mujeres trabajadoras en España: Puntos significativos", Mujer Trabajo y Seguridad Social, La Ley, Grupo Wolters Kluwer, 2012. 
estancamiento de cifras de participación de las mujeres, sobre todo en los ámbitos universitarios se reduzca, y volvamos a posiciones de décadas pasadas donde las mujeres tenían menos opciones de participar en el sistema educativo, sobre todo como estudiantes en las universidades. Este hecho es casi una incógnita por ahora.

\subsection{DOCENCIA EN FEMENINO: ¿QUÉ PASA ENTRE LOS DOCENTES?}

¿Quiénes ocupan los puestos de dirección o representación en el mundo académico? ¿De qué se ocupan las docentes? El famoso gráfico de tijeras, o el actual $18 \%$ de mujeres catedráticas de universidad, ya no es un dato de diferenciación con el significado que tenía en décadas pasadas. Hoy es más importante ser cabeza o director/a de un grupo de investigación que articule niveles de excelencia y posibilidad de acceder a publicaciones JCR o equivalentes, así como a la investigación de mayor demanda, o éxito académico.

El número de mujeres que ejercen de profesoras en el conjunto de enseñanzas representa el $64,8 \%$ en el curso $2010-11$. Este porcentaje ha ido aumentado en los últimos años; ha pasado del 64,0\% en el curso 2008-09 al 64,4\% en el curso 2009-10. Esta cifra llega al 65,2\% en el curso 2011-2012.

Los porcentajes de mujeres en el profesorado según la enseñanza que imparten son especialmente elevados en Educación Infantil y Primaria (82,1\%), en Educación Especial $(81,3 \%)$ y en Enseñanza de idiomas $(74,6 \%)$. Así mismo, el porcentaje más bajo en el profesorado femenino corresponde a Educación Universitaria $(37,2 \%)$ pero la participación femenina es diferente según las categorías de profesorado universitario.

En todos los tipos de profesorado de educación universitaria en las universidades públicas hay mayor participación masculina. La participación femenina más numerosa, según datos del 2010-2011, corresponde a los titulares de escuela universitaria $(41,6 \%)$, titulares de universidad $(38,9 \%)$, y a otro personal docente $(40,4 \%)$. La participación femenina más baja, como ya se ha señalado, corresponde a catedráticos de Universidad (18,1\%). Sólo un curso posterior, 2011-2012 ${ }^{17}$ las catedráticas de universidad ascienden al 19,5\%, y los titulares de universidad pasan al $39,3 \%$. Los gráficos 1 y 2 explicitan esa situación.

Una vez más, son las mujeres las que ejercen las tareas de cuidado y atención de los más pequeños, así como de los más débiles y/o desvalidos, es decir en la educación especial. Y esto no es una cuestión de actualidad. Estos niveles educativos discurren de esta forma desde hace décadas. Pareciera como que lo más laborioso, complicado o

${ }^{17}$ INE, Mujeres y hombres en España/Educación (actualizado 17 marzo 2014). Según los datos de este curso la proporción de mujeres en educación infantil se eleva al 97,5\%. Posiblemente este dato sea una rectificación de la contabilización estadística anteriormente utilizada. 
de mayor dificultad se les reserva a las mujeres. La cosa cambia cuando se trata de otro mercado educativo como es el universitario.

Las cifras son elocuentes si se observa el Gráfico 2. Las tasas de participación del profesorado no universitario femenino presentan unas diferencias, poco significativas entre la enseñanza pública, $70,2 \%$ de mujeres y la privada que asciende al $72,1 \%$. De igual modo, para el curso 2011-2012 aparece una figura, la de personal contratado, en la que figura una cifra del $41,5 \%$ para las mujeres, e igualmente del $21,6 \%$ entre el personal emérito.

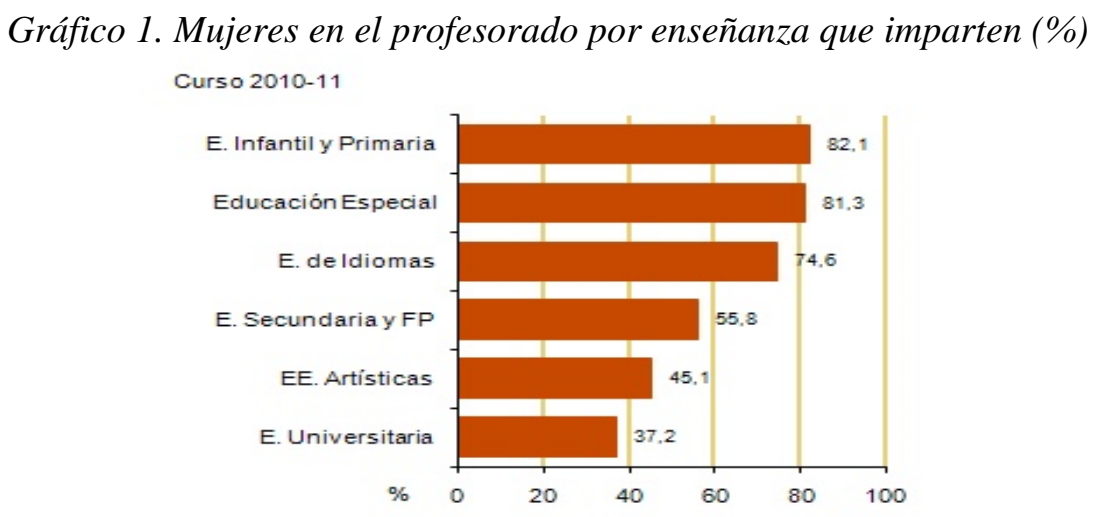

Gráfico 2. Mujeres en el profesorado de educación universitaria en las universidades públicas por categoría (\%).

Curso 2010-11

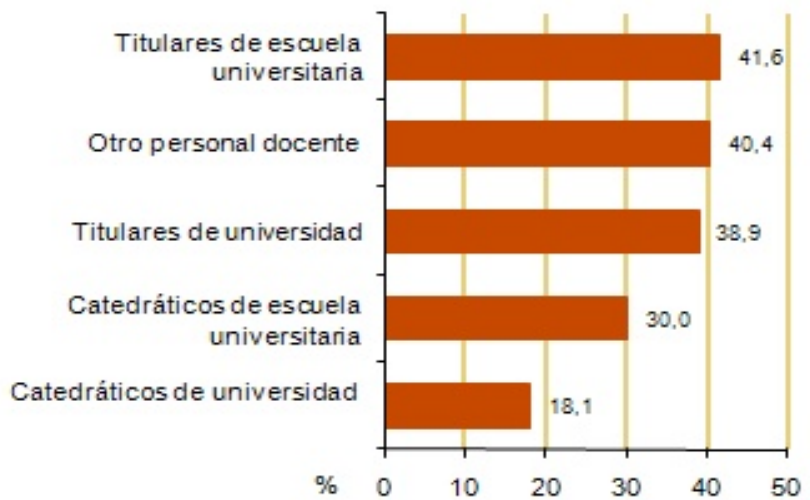

Fuentes: Las cifras de la educación en España. Estadísticas e indicadores. Edición 2013. Ministerio de Educación, Cultura y Deporte. Las cifras de la educación en España. Estadísticas e indicadores. Edición 2013. Ministerio de Educación, Cultura y Deporte Instituto de Evaluación. Sistema estatal de indicadores de la educación. Ministerio de Educación, Cultura y Deporte Estadística de la Enseñanza Universitaria en España. INE Estadísticas de Educación y Formación. Eurostat. 
En la actualidad los cambios sufridos por parte del profesorado universitario, al desaparecer, por un lado la clasificación de las enseñanzas en diplomaturas y licenciaturas, y de un modo alternativo, los conocidos como titulares y catedráticos de escuelas universitarias, para formar dos categorías docentes: titulares y catedráticos, a los que a lo sumo les separan años de docencia, la situación debería de ser de mayor igualdad en la distribución por sexo-género de estos dos tipos de docentes, pero este es un proceso todavía lento en cuanto al número de componentes mujeres en cada nivel o categoría de enseñantes.

\subsection{RESULTADOS EMPÍRICOS CONTRASTABLES: DIFERENCIAS EN ACREDITACIONES Y SEXENIOS}

Uno de los aspectos que en la docencia se convierte en relevante son las diferencias entre acreditaciones y sexenios obtenidos por parte de los docentes. Los méritos adquiridos son evaluados y poseen un nivel de prestigio así como de poder que se traduce en significativas diferencias entre el profesorado. Los que pueden dedicar más tiempo a la docencia y sobre todo a la investigación pueden publicar más y obtener unos mejores resultados para acceder a las acreditaciones y también para acumular sexenios.

Los resultados de las acreditaciones por rama y sexo para los catedráticos de universidad se muestran en la Tabla 1, y aparecen diferenciados no sólo por rama de conocimiento, sino también por sexo. De igual modo, el Gráfico 3 permite apreciar que el porcentaje de evaluaciones positivas es ligeramente más elevado entre los hombres que entre las mujeres en seis de las diez comisiones de evaluación -mientras que el caso contrario, más mujeres acreditadas, sólo se da en dos de las diez comisiones, como se puede ver en la Tabla 1 -CU Ciencias de la Salud y CU Ingeniería y Arquitectura-. En concreto, destaca el porcentaje de mujeres acreditadas como catedrático en ciencias de la salud (77\% frente al $73 \%$ de hombres), lo que puede evidenciar la existencia de una bolsa de mujeres cualificadas que no habían podido promocionarse con los sistemas anteriores. Por el contrario, en Ciencias sociales y jurídicas destaca la desproporción de acreditaciones, $59 \%$ en el caso de las mujeres y $64 \%$ para los hombres. Es significativo que en Ciencias $(83 \%)$ hay una igualdad en el número de participantes hombres y mujeres.

Tras la consulta y reflexión de los datos analizados más arriba que ese $65 \%$ de mujeres que ejercen como profesoras en el total del sistema educativo es una fuerza productiva importante. En apariencia el mundo del trabajo productivo asalariado se divide en una vista rápida entre la empresa y los servicios públicos, fundamentalmente el mundo de la educación y el de la salud. Y es en este último donde la participación femenina está consolidándose. Tareas que son dificultosas, que conllevan el riesgo de 
la invisibilidad ${ }^{18}$, por ejercerse de puertas para dentro, como el caso de profesionales del sector de la educación y de una parte del de salud, requieren más de paciencia, de sacrificio, y en muchos casos de renuncia. Suelen ser trabajos peor pagados y el reconocimiento social en términos de prestigio es menor; también las condiciones materiales y el apoyo humano es bastante inferior. Son las mujeres que han llegado más tarde a realizar trabajos especializados, que deben o pueden efectuarse fuera del hogar, y que a menudo son compatibles con el trabajo doméstico, pues pueden llevarse a cabo sin el riesgo de estar compitiendo por el mejor resultado, es decir por la obtención de éxitos de mercado, compitiendo con otros compañeros; y por lo mismo pueden ajustarse a tiempos marcados por el reloj, es decir están sujetos a horarios fijos. Son precisamente algunas de esas mujeres trabajadoras, las que tienen ese singular proceso de adaptación y constancia, las que dominan ese sector.

Tabla 1. \% de profesores acreditados como catedráticos según sexo, y rama de conocimiento, 2012

\begin{tabular}{|l|r|r|r|}
\hline & \multicolumn{1}{|c|}{ Mujer } & \multicolumn{1}{c|}{ Hombre } & \multicolumn{1}{c|}{ Total } \\
\hline $\begin{array}{l}\text { ARTES Y } \\
\text { HUMANIDADES }\end{array}$ & $71 \%$ & $73 \%$ & $72 \%$ \\
\hline CIENCIAS & $83 \%$ & $83 \%$ & $83 \%$ \\
\hline $\begin{array}{l}\text { CIENCIAS DE LA } \\
\text { SALUD }\end{array}$ & $77 \%$ & $73 \%$ & $74 \%$ \\
\hline $\begin{array}{l}\text { CIENCIAS } \\
\text { JUCIALES Y }\end{array}$ & $59 \%$ & $64 \%$ & $62 \%$ \\
\hline $\begin{array}{l}\text { INGENIERIA Y } \\
\text { ARQUITECTURA }\end{array}$ & $70 \%$ & $69 \%$ & $69 \%$ \\
\hline TotaI & $\mathbf{7 2 \%}$ & $\mathbf{7 2} \%$ & $\mathbf{7 2 \%}$ \\
\hline
\end{tabular}

Algunos de esos trabajos, el de enseñantes en primer lugar y también el de cuidadoras de salud los que más se parecen a las tareas "más nobles" de cuidados en el hogar. La revolución callada, silenciosa es que son trabajos, muchos de ellos, que no exigen brillantez, competitividad, incluso se pueden hacer en solitario, aunque estén basados en la cooperación. Pueden incluso ser realizados aceptando un jefe que decida, basta con ejecutar calladamente, y seguir las órdenes del que dirige, que en muchas ocasiones es hombre, que organiza, maneja espacios, decide la agenda del día, y organiza el trabajo de grupos diversos, ayudado de teléfonos, ordenadores y secretarias. Tiene el poder de decisión pero no la posibilidad de ejecución. Esa persona, revisa, controla, cambia el modelo de lo que debe hacerse, etc. Evidentemente tiene prestigio y poder, pero en muchas ocasiones no sabe ejecutar,

${ }^{18} \mathrm{M}^{\mathrm{a}}$ Ángeles Durán, explica ese tipo de costes, así como el precio del trabajo en el cuidado de la salud, $\mathrm{M}^{\mathrm{a}}$ Ángeles Durán, Los costes invisibles de la enfermedad, Bilbao, Fundación BBVA,2002, pág. 109 y ss. 
porque no se ha entrenado para esa tarea. Pienso en los centros de educación como en los de salud, sea el nivel que sea sobre el que nos centremos. Probablemente el cambio más decisivo es en este caso, será el poder transformar la visión que tenemos sobre la importancia de esa manera nueva de incorporarse al trabajo. $\mathrm{Y}$ en último caso en la decisiva importancia del oficio profesional de las mujeres.

En el Gráfico 3 se recogen los resultados de las evaluaciones de los profesores solicitantes a concursos de acceso. En este ámbito el número medio de sexenios tanto de los acreditados como no acreditados al cuerpo de catedráticos, por rama y sexo es decisivo. En dicho gráfico se aprecia que, en líneas generales, las mujeres tienen unos resultados (\% de evaluaciones positivas) un poco menor que sus compañeros varones. Destaca, no obstante, el caso de ciencias de la salud, en donde los grupos aparecen empatados. Por el contrario, en artes y humanidades e ingeniería y arquitectura la situación es mucho más favorable para los hombres que para las mujeres.

Gráfico 3. \% de solicitantes de sexenios por rama de conocimiento y sexo (2012)

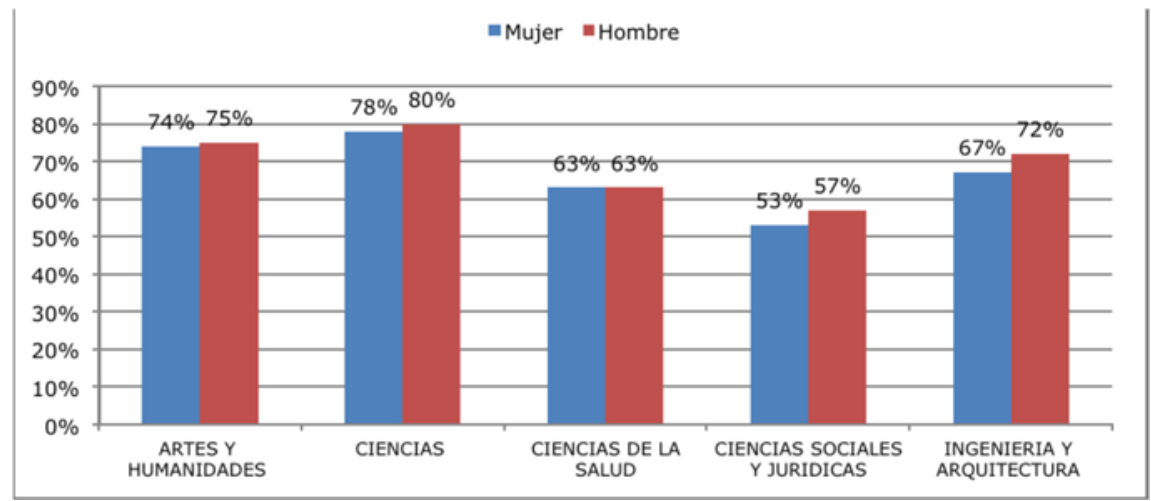

Las diferencias en relación con los acreditados dentro de las ramas se convierte en más notables y la diferencia en solicitantes respecto al sexo en Ciencias de la Salud desaparece, de forma que en Ciencias de la Salud se acreditan la misma proporción de mujeres que de hombres o en Artes y Humanidades, que hay un punto de diferencia a favor de los hombres; mientras que en el caso de Ingeniería y Arquitectura la diferencia es de 4 puntos porcentuales (67\% mujeres y $72 \%$ hombres), y también en Ciencias sociales y jurídicas, un $53 \%$ de mujeres y un $57 \%$ de hombres. Las diferencias de acreditación son significativas sobre todo si se tiene en cuenta que la proporción de evaluaciones positivas es significativamente más alta en Ciencias, 78\% a favor de las mujeres y $80 \%$ hacia los hombres.

Aquí es preciso hacer dos lecturas. Por una parte el prestigio y tradición de lo que es científico, que plantea la presencia de supuestos en los que las evaluaciones podrían valorar a la baja aquellas disciplinas cuyos presupuestos no son tan perfectamente cuantificables o medibles como parecen serlo, al menos en la percepción de muchos profesores ocupados de las tareas de evaluación. La tradicional 
y obsoleta división de los saberes en Ciencias y Humanidades, incluyendo aquí las Ciencias sociales y jurídicas, podría ser una tendencia negativa para las mujeres, puesto que bastantes estudios califican la inteligencia y actitudes de las mujeres más volcada al segundo tipo de saberes, las humanidades. La otra explicación más directa es que los modelos de evaluación aplicados y la valoración de los méritos están calculados y conducidos desde las ciencias básicas. Los baremos que se suelen utilizar centran su atención sobre todo en los modos de producción del conocimiento científico propio de las ciencias experimentales, la medicina o la ingeniería.

\section{MERCADO PROFESIONAL Y SUS EXCLUSIONES}

El mundo empresarial va a actuar con otras claves y, a pesar de que se han producido avances, sigue siendo "cosa de hombres". Así valoraba el periódico El País en Mayo del 2013 en una editorial dedicada al apartado de Negocios ${ }^{19}$ y planteaba el controvertido discurso de la necesidad de las cuotas, "en tanto en cuanto esa fórmula puede ser el mal menor mediante el que se garantice una relativa proporcionalidad" en relación con la presencia de las mujeres en las empresas "cotizadas" ${ }^{20}$. La "normalización" no se traslada a los puestos de mando, y de hecho a medida que se sube en la escala laboral aparece un menor número de mujeres. En un reciente estudio, patrocinado también por El País se señalaba que de más de siete mil directivas estudiadas, sólo un $22 \%$ ocupaba esos encargos; por otra parte, el $11 \%$ lo hacía en puestos de alta dirección, y el $10 \%$ eran consejeras ${ }^{21}$.

La previsión de la Ley de Igualdad y las recomendaciones de Bruselas no han sido suficiente acicate, y el resultado es una clara desigualdad de género. De este modo, sólo tres de 114 empresas cumplirían hoy la Ley de Igualdad. No obstante es indicativo que el artículo del diario El País señale que son las propias mujeres las que limitan su expansión o ponen pegas a las propias mujeres. Carlos Alemany ${ }^{22}$ indica ese

19 "La incorporación de las mujeres a los puestos directivos de las empresas españolas es un proceso tan lento que bien podría considerarse como un fracaso social. La decepcionante evolución del ascenso femenino a puestos de alta dirección puede cuantificarse, pero las explicaciones suelen ser confusas o insatisfactorias" El País, 12 de Mayo de 2013, pág. 3

20 Según el CEC (Congreso Empresarial para la Competitividad) la incorporación de las mujeres en las empresas ha experimentado un cambio importante en el caso de España desde la transición, si bien a pesar de los esfuerzos sólo el 37\% de las plantillas de los 114 grupos analizados son mujeres.

${ }^{21}$ Ese mismo estudio indicaba que Joan Rosell, presidente de la CEOE, admitía el fracaso de promover la presencia femenina en las empresas y concluía que ha tenido que tirar la toalla en su objetivo de que en los estatutos de la organización figurase que el $25 \%$ de la representación patronal correspondiera a mujeres

${ }^{22}$ Socio director de Alemany\&Partners, Carlos R. Alemany funda una nueva firma de búsqueda de directivos, que tiene como objetivo posicionarse como un referente en este sector. Alemany cuenta con una amplia experiencia en la búsqueda y valoración de personas para la alta dirección. 
defecto, y concluye que son las propias mujeres que ocupan puestos de mando, ya que creen que a ellas nadie les ha ayudado y están en esos puestos por sus propios méritos. Prefieren que el grupo de mujeres que aún no ha llegado a ese rendimiento, tenga las mismas dificultades que tuvieron ellas.

En esta misma línea otros estudios anteriores, promovidos desde el Instituto de la Mujer, centrados en el poder y la participación de las mujeres en el mundo empresarial, relataban una situación parecida. Bajo la dirección de Cecilia Castaño se realizó una investigación en 2008 , sobre la visibilidad y poder en las empresas ${ }^{23}$. Un parte de la investigación estaba basada fundamentalmente en la explotación de los datos de la EPA de 2006. Entre los temas que resalta este estudio está la asociación de las categorías profesionales. Aunque parece bastante obvio, son las mujeres con mayor nivel de estudios las que aparecen como empresarias: un $43,7 \%$ tiene estudios superiores y sólo un $10 \%$ de ese grupo empresarial tiene sólo educación primaria. Por otra parte de las que tienen educación superior sólo un 37,5\% son directivas, mientras que el caso de los varones la cifra de los directivos con educación superior sube al $45 \%$.

\section{BREVE APUNTE SOBRE EL OFICIO DE SOCIÓLOGO O DE POLITÓLOGO: SOCIÓLOGAS, GÉNERO Y FEMINISMO}

La profesión sociológica se muestra, en apariencia, como fuera del ángulo de profesiones consolidadas, asentadas en el mercado de trabajo, con papeles y status reconocidos, y una parte de ellas con una historia bien definida. Sin duda, parte de esas peculiaridades, se deben, como indican algunos de sus "creadores" o introductores de sus saberes, a la especial "misión" de ser la pieza crítica del conocimiento en ciencias sociales. Hoy en día esta profesión se debate entre la neutralidad postulada por alguno de sus clásicos, Durkheim sería uno de sus representantes, o bien por el arriesgado papel que le otorgan otros de sus actuales teóricos de la sociología contemporánea. El debate interno más singular se presenta entre los llamados sociólogos, analistas críticos de la realidad social, y los especializados en los hechos políticos, o especialistas en la realidad política. En la situación actual este hecho permite numerosas interpretaciones, dado que en cierta manera los comportamientos y realidades sociales plantean un devenir diferente y a veces desigual y contradictorio a ese otro nivel llamado el contexto político. Las dos caras de la realidad propondrían un "deber ser" cercano a la norma o a lo jurídico,

\footnotetext{
${ }^{23}$ Instituto de la Mujer, Ministerio de Igualdad, Mujeres y poder empresarial en España, Madrid, 2009. El estudio según planteaba su investigadora principal tenía un finalidad exploratoria: "Los objetivos de la investigación son profundizar en el conocimiento de las características de las mujeres que participan en la toma de decisiones en las empresas, sus actitudes, las implicaciones de los estilos de dirección de mujeres y hombres desde una perspectiva de género, así como el efecto de la presencia de mujeres en los puestos de máxima responsabilidad sobre los resultados económicos de las empresas"
} 
mientras que los hechos sociales como realidades cambiantes poseen la característica de ser dinámicos, y en algunas ocasiones difícilmente observables y cuantificables.

Todo ello plantea dos modelos distintos de construir el espacio propio de actuación de sus profesionales. Los sociólogos se supone que observan, describen y tipifican una realidad, mientras que en el segundo caso la realidad social necesita de etiquetas valorativas de las referencias analíticas que desde la política se propongan. Así la ideología política, o el partido político al que pertenece cada persona, o la intención de voto, serían indicadores más frágiles para ser contabilizados. Dadas esas peculiaridades se produce una curiosa experiencia: la inseguridad de los primeros con el tema de ser los más "críticos analistas sociales" y la osadía de los segundos sentenciando las políticas sociales y cualquier plan que pueda ponerse en marcha por algún tipo de institución social o política. De igual modo, se registra la falta de credibilidad de los sociólogos como profesionales por la débil preparación en métodos y técnicas, así como por el uso de nuevas tecnologías y el desmedido afán de teorizar. Su status y papel social en general nos lleva a reflexiones frecuentes de quiénes somos ¿cuál o cuáles son nuestras funciones? ¿Para qué servimos? Un estudio reciente patrocinado por la ANECA situaba la profesión de sociólogo/a con sus dos derivaciones como sociólogos y politólogos, si bien existe una ligera ventaja en la inserción profesional en el ámbito de la politología, en torno a un $60 \%$ ejercían su profesión como técnicos de la administración pública, consultor de recursos humanos, o técnico en programas sociales o bien docentes.

Las mujeres en las aulas, cuya presencia ha crecido en la mayoría de los centros dedicados a este tipo de estudios, no aparecen en datos de tanta importancia y envergadura como es el caso citado anteriormente patrocinado por ANECA, que no se plantea la necesidad de diferenciar esta situación, ni siquiera por curiosidad. A modo de ejemplo, una institución, el IBPP (Instituto de Bienes y Políticas Públicos) de reciente creación, cuyos especialistas más importantes parecen ser politólogos o sociólogos, tiene en la actualidad una investigadora científica, como categoría más alta entre su personal. De igual modo hay 6 científicas titulares, y 7 científicos titulares. A partir de aquí hacia abajo la pirámide invierte su composición y entre el personal contratado y becario, hay 10 mujeres frente a 2 varones. No hay ninguna mujer en el ámbito de la dirección ${ }^{24}$.

Aunque son pocos los datos existentes sobre la participación de mujeres en centros de investigación o en empresas de sector de servicios o tecnología, los y las sociólogas se distribuyen en el mundo profesional con nuevos papeles, en las ONG`s, y en el campo de los servicios públicos a nivel de la administración, sobre todo local. Participan en diversos campos tales como el militar, la protección civil, el tema de riesgos, o las crisis y catástrofes, o desastres naturales; también a nivel de recursos humanos, en la responsabilidad social corporativa, o en el diseño de programas municipales entre otros. Hay algunas salidas que aún no se han producido, dada la

${ }^{24}$ Datos facilitados por una de las investigadoras del centro en conversación telefónica 
novedad de la crisis, y las nuevas situaciones que se pueden derivar de esos cambios. Algunas de las incógnitas que se pueden plantear siguen a continuación:

1. ¿Esta crisis se resolverá como otras, con un proceso evolutivo, de estallido, crecimiento o progresión, estabilización, decrecimiento y cierre de ciclo?

2. El proceso y sobre todo el resultado de esta crisis parece no obedecer al patrón anterior, debido a que su carácter es de naturaleza sistémica, es decir, se han convulsionado las estructuras así como las relaciones entre las mismas.

3. Se aprecia que los sociólogos/as no están trabajando en estos campos ¿Cuál sería su visión anticipada de la nueva sociedad post-crisis? ¿Cuál sería el papel de las mujeres sociólogas en el estudio y análisis de la misma, con sus propias reflexiones desde la perspectiva de género?

\section{CONCLUSIONES Y SUGERENCIAS}

Las tesis de diferentes estudiosos del tema es que la igualdad no es cuestión solo de leyes, datos y cifras del avance de la participación de las mujeres en la sociedad actual, ¿tuvo este hecho alguna consecuencia en términos de retroceso para el bienestar de la sociedad? En el caso de España se señala el efecto de un espectacular descenso de la natalidad. Según algunos expertos las mujeres nos hemos dedicado a más tareas de "las que nos estaban encomendadas" y esto ha dado lugar a una caída espectacular de la natalidad, que junto a otros factores ha permitido que estemos, $o$ hayamos estado en caída constante, también en este ámbito; es decir por debajo del índice de reemplazo. Es una cadena, si nacen menos niños, si emigran los más preparados, si aumenta la esperanza de vida de los adultos, si hay menos afiliados a la Seguridad Social, ¿cómo vamos a poder cuidar a nuestros mayores, y sobre todo quién va a poder hacerlo?, ¿podrá resultar eficaz la más notable la diferencia en preparación mayor de nuestros jóvenes con respecto a dos décadas pasadas si el panorama más cercano es cuidar a los más mayores?, y, sobre todo, ¿qué pasará con una parte de esas mujeres que con muy buena preparación se pueden ver obligadas sólo a cuidar a los más mayores y por tanto no podrán hacer otras cosas que sean más atractivas para su vida personal?

Bastantes tareas de las que se ocupan las mujeres, sobre todo en el hogar no las realizan muchos hombres ¿Son diferentes o superiores las mujeres? En apariencia si se revisan las cifras estadísticas que tienen en cuenta el rendimiento educativo nos encontramos que las mujeres obtienen el título de ESO en el 36\% de los casos, frente a sólo el 18\% de varones. En los índices de fracaso escolar, hay 10 puntos de diferencia a favor de las mujeres (21\%, frente al $31 \%$ de varones). Existe sin duda una "disarmonía" favorable a las mujeres, que parece no tener fácil solución. Seguimos por tanto en un camino que nos hace desiguales, a lo mejor como no esperábamos, pero a la larga, salvo excepciones con mejores resultados, incluso a pesar de las desigualdades. Luchemos desde la mejor preparación que hemos llegado a conseguir 
como grupo para hacerlas desaparecer.

\section{REFERENCIAS BIBLIOGRÁFICAS}

ALMARCHA, AMPARO (2013) “Aquellos días de Serrano 19”, en García Sanz, B., Iglesias de Ussel,; Oltra, B., Martinez Paricio, J., Valles, M., De la sociedad española y otras sociedades. Libro Homenaje a Amando de Miguel, Madrid: CIS.

DOMINGUEZ, CARMEN (2002) El futuro de la conciliación entre la vida laboral, familiar y personal: Mujeres, trabajo y calidad de vida. Mimeografiado.

DURÁN, Ma ÁNGELES (2002): Los costes invisibles de la enfermedad. Bilbao: Fundación BBVA.

INE (2011) Informe Hombres y mujeres.

INSTITUTO DE LA MUJER (2009): Mujeres y poder empresarial en España. Madrid: Ministerio de Igualdad.

MADOO, PATRICIA y NIEBRUGGE-BRANTLEY, JILL (1993): "Teoría Feminista Contemporánea" (pp. 353-409), en George Ritzer, Teoría Sociológica Contemporánea. Madrid: McGraw-Hill.

MIGUEL, AMANDO DE (2010): Memorias y desahogos. Madrid: Infova Ediciones.

MILLER, LUIS M. (2004): Participación laboral y Estados de bienestar, REIS, 108:49-74

PERDOMO, INMACULADA y PUY, ANA (2012) Género, conocimiento e investigación. Madrid: Plaza y Valdés.

TOBIO, CONSTANZA (2002): Conciliación o contradicción: como hacen las madres trabajadoras, en REIS, 97: 155-186. 